had arisen, 4\% reported that they had run out of kits and $2.7 \%$ said there was confusion when signing the kits out of the controlled drug $(\mathrm{CD})$ register.

Three weeks out of 25 saw all the kits being used, average usage is 4 intubation kits per week. 97.4\% reported the doses used were effective in sedating and paralysing the baby prior to intubation, $2.6 \%$ commented that they were somewhat effective but that in one occasion the paralysis had not been optimal, however they questioned whether the cannula had been functioning properly.

Conclusion The implementation of ready to use intubation drug kits has made the process of preparing for an intubation easier and quicker for all involved in the process. Having the dose banding set up on the electronic prescribing system has reduced the chance of prescribing errors and the pre- filled kits have reduced the chances of calculation errors during drug preparation. When the kits run out there are instructions in the guideline detailing how to make the required concentrations. As a result of this study standardised teaching videos were introduced from the beginning of July 18. Further simulations have been completed to ensure that all staff follow a standardised process. Next steps are to ensure that the documentation in the $\mathrm{CD}$ register includes all necessary information without any need for amendments. To overcome this, a stamp is being designed to use in the book each time a patient requires a kit, thereby providing a prompt for the nurses.

\section{P048 THE EFFICIENCY OF AN ELECTRONIC PRESCRIBING SYSTEM ON CLINICIAN'S PRESCRIBING THROUGH THE PHARMACIST'S REVIEW FUNCTION}

Yusuf Asif, Amrita Garcha. Birmingham Women's and Children's NHS Foundation Trust

\subsection{6/archdischild-2019-nppc.57}

Aim To assess the efficiency of an electronic prescribing system (EPS) on clinician's prescribing through the implementation and use of the pharmacist's review function.

Introduction An electronic prescribing system, PICS, was launched in one ward, the liver unit, in April 2017. Many features were available on the EPS to support safer prescribing such as clinical decision support and prescribing guidance. One particular feature was the review note function, which was available for pharmacists, to attach a review note to a selected drug, highlighting an intervention, in order for the clinician to review. Once a review note was added, an eye icon appeared next to the selected drug on the drug chart. Once the prescription was reviewed, it could be signed off to signify the note was acknowledged and actioned. Implementing the review function, pharmacists are guiding safer prescribing of clinicians based on their clinical knowledge and expertise.

This is evidenced by the Royal College of Physicians who have highlighted how healthcare professionals should support each other on the safer use of medicines, ${ }^{1}$ and The Royal Pharmaceutical Society, that stated the pharmacy team provides expertise and advice to support the safe and effective use of medicines by patients'. ${ }^{2}$

Methods An audit was completed, over a two-week period, to assess the type of interventions, the timeliness in which the intervention was noted once the prescription was added and the timeliness in which the clinicians reviewed and actioned a prescription which had a review note attached to it by the pharmacist. Pharmacists annotated all interventions using the review function on PICS. The date and time when the prescription was added, the review note was added and the review note was signed off was recorded. The type of review note (intervention) and the change made, if made, was also noted. It is recognised that there will be a delay from when the pharmacist adds the review note and the clinician views it. For urgent reviews, the clinician was verbally notified.

Results 29 interventions were recorded over the two-week period, with the majority of interventions involving dosing issues (41\%), followed by interventions regarding formulation (17\%), drug and frequency (both 14\%). Most review notes were added 24 hours (34\%) after the prescription was added onto the patient's drug chart followed by those noted within an hour $(21 \%)$ of the prescription being added. 25 prescriptions (86\%) were amended upon the advice of the pharmacist whilst 4 prescriptions (14\%) were not, due to a clinical requirement or if the patient had been discharged. 21 review notes $(72 \%)$ did not require the pharmacist to verbally inform the clinician to amend the prescription.

Conclusion The audit highlighted the importance of the pharmacist's review function in highlighting interventions, whether this was related to dosing, formulation, frequency or drug. In addition, it highlighted the value of the pharmacist's interventions via the review function as most review notes were amended as per the pharmacists' advice and the majority did not require verbal notification to the prescriber, stressing the importance of the function.

\section{REFERENCES}

1. Supporting safe prescribing. Royal College of Physicians 2017.

2. Royal Pharmaceutical Society. Professional standards for hospital pharmacy services: optimising patient outcomes from medicines. London: RPS, 2014.

\section{P049 PHARMACIST 5PS- POSITIVE PRAISE PRODUCES PLEASING PRESCRIBING}

Aimee Cope, Adrian Plunkett, Alison Jones, Alice Chan, Katie Price, Rhian Isaac. Birmingham Women and Children's NHS Foundation Trust

\subsection{6/archdischild-2019-nppc.58}

Aim Learning from Excellence (LfE), a positive reporting initiative, has two main objectives: to capture and learn from episodes of excellent practice and boost morale through positive feedback. The PRAISe project, ${ }^{2}$ tests the hypothesis that positive reporting and appreciative inquiry (AI) can be used as interventions to facilitate behavioural change and improvement in antimicrobial stewardship.

Methods LfE was applied as a quality improvement (QI) intervention for antimicrobial use on PICU over a 12 month period: baseline ( 3 months), intervention (6 months) and post intervention (3 months) phases. 31 PICU charts were screened weekly by PICU research nurses, this included any documentation added by a pharmacist to improve antimicrobial stewardship. Positive reports (IR2) were generated for gold standard prescriptions and excellence in antimicrobial stewardship, followed up by AI. QI suggestions derived from AIs were applied to the antimicrobial stewardship programme of the unit e.g. RAG rating antibiotics to the prescription charts. PICU pharmacists recorded interventions relating to antimicrobials during the data collection period. Pharmacist interventions were split into proactive or reactive: proactive involving 